\title{
I silenzi di Alatiel
}

\section{Laura Benedetti}

Gli studi sul ruolo della differenza sessuale nel linguaggio si sono molto sviluppati negli ultimi vent'anni. L'idea del linguaggio come trasparente veicolo del pensiero è stata soppiantata da un'idea diversa, discriminante e attenta alle sfumature sociali e di gender. Si è evidenziato così che il linguaggio costringe ed inibisce almeno tanto quanto esprime, che intere tranches de vie, non solo femminili, sono state consegnate al silenzio per mancanza di parole che potessero veicolarle; si sono riletti i miti per capire come, nel momento in cui Adamo ha preso possesso del mondo assegnando un nome agli animali e alla donna, ad Eva è stato concesso un accesso al mondo secondario, attraverso un vocabolario compilato da altri.

In questa esperienza di distanza dalla lingua, trovano spazio vie di fuga ...: il silenzio, il residuo non detto, il corpo piuttosto che il pensiero.... Se io sono il linguaggio dell'altro, decido di negare questa estraniazione negando me stessa; piuttosto che dirmi in un linguaggio straniero, allora il silenzio. Simbolo impotente, perché nel silenzio ancor meglio mi parlo e mi penso sempre all'interno di quella rete concettuale che ha suoni da me non profferiti. Nel silenzio tace il suono, non la parola. (Cavarero 53)

Introdurre, come fa Cavarero, una distinzione tra suono e parola, significa porre l'accento sul linguaggio come attività conoscitiva, rifacendosi in maniera implicita al logos greco, insieme parola e ragione. Il silenzio non è dunque, di per se stesso, ribellione e garanzia di estraneità alle logiche altrui. Il silenzio non è neutro ma offre più spazio all'interpretazione di quanto non faccia il suono. Bisogna, come scrive Irigaray,

questionner le fonctionnement de la "grammaire» de chaque figure du discours, ses lois ou nécessités syntaxiques, ses configurations imaginaires, ses réseaux métaphoriques, et aussi, bien sûr, ce qu'elle n'articule pas dans l'énoncé: ses silences. (73)

"Questionner ... les silences" potrebbe ben fungere da epigrafe a queste note sulla novella di Alatiel (Decameron 2.7).

Se si legge il Decameron con un'attenzione specifica rivolta al manifestarsi della differenza sessuale nel linguaggio, non si può non rimanere sorpresi dalla lucidità con cui Boccaccio formula i termini del problema: le donne, per lui, hanno senza dubbio col linguaggio un rapporto precipuo, diverso, in ogni caso, da quel- 
lo degli uomini. In particolare, l'importanza, per la donna, della risposta pronta, del motto arguto, viene sottolineata in modo implicito in quelle novelle in cui la protagonista sbroglia una situazione $(1.5,1.9,6.7)$ o addirittura si salva la vita (6.7) grazie ad una frase opportuna tempestivamente detta.

In altri casi, ci troviamo di fronte ad argomentazioni distese ed esplicite, come questa di Pampinea che introduce la novella conclusiva della prima giomata:

Valorose giovani, come ne' lucidi sereni sono le stelle ornamento del cielo e nella primavera i fiori ne' verdi prati, così de' laudevoli costumi e de' ragionamenti piacevoli sono i leggiadri motti; li quali, per ciò che brievi sono, molto meglio alle donne stanno che agli uomini, in quanto più alle donne che agli uomini il molto parlare e lungo, quando senza esso si possa far, si disdice, come che oggi poche 0 niuna donna rimasa ci sia la quale o ne 'ntenda alcun leggiadro o a quello, se pur lo 'ntendesse, sappia rispondere . . queste così fregiate, così dipinte, così screziate 0 come statue di marmo mutole e insensibili stanno o sì rispondono, se sono addomandate, che molto sarebbe meglio l'aver taciuto. (1. 10.3)

Il concetto sarà ribadito con parole quasi identiche da Filomena nell'introduzione alla novella di madonna Oretta (6.1). Vale la pena di notare la posizione strategica del passo, che compare alla fine della prima giornata per essere ripreso esattamente a metà opera. L'uso del linguaggio da parte della donna, e da parte di uomini di condizione sociale inferiore a quella dei loro antagonisti, ha spesso una funzione decisiva e finisce per costituire un surrogato dell'azione. Ma il passo citato è interessante anche per un altro motivo: l'espressione "come statue di marmo mutole" combina infatti elementi che ritornano, scissi, in due novelle il cui elemento decisivo non è la parola, ma il silenzio della donna. Così infatti protesta Zima:

... voi mi prometteste di farmi parlar con la donna vostra, e voi m'avete fatto parlar con una statua di marmo. (3.5.26)

Analoga recriminazione viene rivolta a messer Gentile:

Messere, bella cosa è questa vostra, ma ella ne par mutola. (10.4.34)

Nell'ultimo caso, l'“esser cosa" è in stretta relazione con l'"esser mutola": l'accento si sposta, con più decisione, sulle conseguenze disumanizzanti dell'assenza della parola. Il discorso sul linguaggio fa così intravedere in filigrana quello sul suo contrario, e l'abilità dialettica di tanti personaggi femminili del Decameron fa da contraltare alle peripezie di quelli cui la parola è stata interdetta da un'autorità o dalle circostanze. Bisogna infatti tener presente che il silenzio della donna non è presentato come scelta, ma ha una giustificazione narrativa precisa nell'economia della novella. A volte è l'imposizione maritale a sbarrare la strada al linguaggio; in altri casi la donna rimane senza parole perché si trova in una zona a lei estranea: la bella Alatiel, vergine promessa sposa del re del Garbo, naufraga in una terra in cui le sue parole non sono più comprese, non servono più. 
La novella di Alatiel vanta una complessa tradizione esegetica: generazioni di critici si sono cimentati con l'insondabile silenzio della protagonista. La struttura stessa della novella si ribella ad un'interpretazione univoca. Già il prologo di Panfilo presenta scarti logici notevoli: la prima parte sembra stigmatizzare il comportamento degli amanti di Alatiel, mentre la seconda adatta la regola generale (non desiderare più di quel che Dio ci ha dato) al caso specifico della bellezza, che le donne non devono cercare di accrescere. Sembra la trita polemica, di derivazione patristica, contro la vanità femminile, ma la storia proposta come deterrente non illustra il precetto che con una certa approssimazione, in quanto Alatiel non si sforza di sembrare più bella, ma è per natura meravigliosa, per cui l'accento si sposta dall'ambizione illegittima alla fatalita ineluttabile. All'introduzione edificante fa poi riscontro sia il commento finale dello stesso novellatore (" "Bocca basciata non perde ventura, anzi rinnuova come fa la luna»" 122), sia la reazione delle donne della brigata che, sospirando forse più per invidia che per pietà delle sorti di Alatiel, non solo sanciscono come fallito il tentativo di educazione, ma decretano eccentrica la novella rispetto al tema della giomata, tema che prescrive di ragionare "di chi, da diverse cose infestato, sia oltre alla sua speranza riuscito a lieto fine". Il sorriso delle donne della brigata implica che Alatiel non è poi tanto sventurata ad aver fatto tante nozze: ma è questa la sventura di Alatiel?

Paradossalmente, se Alatiel incontrasse Antigono quando ancora con Pericone, suo primo possessore, il meccanismo sarebbe più chiaro: la futura regina cade in disgrazia ma la fortuna l'aiuta restituendola al ruolo che le era destinato fin dall'inizio. Di che segno sia la sua sventura è chiaro nel passaggio da Pericone a Marato:

Non essendo la fortuna contenta d'averla di moglie d'un re fatta divenire amica d'un castellano, le si parò davanti più crudele amistà. (31)

L'ambizione sociale è, ancora, il movente della decisione di Alatiel di palesarsi ad Antigono: ella spera, così facendo,

di dover potere ancora nello stato real ritornare. (92)

Il fatto che la perturbazione si ripeta nove volte, otto delle quali accompagnate da un rapporto sessuale, confonde i contorni della novella, che nei commenti di Panfilo e delle donne si trasforma da storia di fortuna in storia di seduzione, mentre il silenzio imposto dalle circostanze alla protagonista lascia il campo aperto alle interpretazioni sulla sua eventuale complicità, o almeno connivenza, con il comportamento dei suoi possessori. Più che riflettere l'interpretazione in chiave comica offerta dalla brigata sul prologo per svelarne il presunto sarcasmo, come altri ha fatto, vorrei rifarmi all'analisi di Mazzacurati, che salvaguarda le tensioni del testo anche a scapito della "totale prescienza ed onniscienza degli esiti . . . a parte auctoris" (36). In particolare, riscontrare ed indagare l'aspetto comico della novella non ne annulla le contraddizioni, perché tale aspetto convi- 
ve con scene di insolita truculenza nel Decameron, quali quella dell'omicidio e ritrovamento di Ciuraci ( 55 e 61). Aspetti tragici e aspetti comici sembrano avere inoltre una radice comune nel meccanismo ferreo che regola la novella, un meccanismo che riduce non solo Alatiel, ma anche gli uomini che le si accompagnano, a fantocci o meglio ad animali, deresponsabilizzati dal carattere fatale dell'attrazione verso Alatiel involontaria assassina: per Almansi quegli uomini

seem to be repeating the zoological phenomenon of the male bees who burn up their existence in a fatal coitus with the queen-bee. (125)

La comicità (ma una comicità amara, e, oserei dire, pirandellianamente umoristica) nasce quando questi esseri danno un segno della propria individualità, per poi essere catturati dal meccanismo indifferente. Penso ai propositi di castità di Alatiel smentiti subito dal suo ballo alessandrino in condizioni di ebrietà, o all'ingenuità di Antioco che affida in punto di morte la sua donna e le sue cose all'amico mercante: questi gli promette che farà dell'una e delle altre quello che crede sia di consolazione alla sua anima. Come se il fuco, nel suo volo verso la regina, facesse voto di castità. Anche in questo caso, i buoni propositi saranno smentiti alla prima tempesta (del Mediterraneo e degli istinti).

La novella si basa sul principio della ripetizione, come risulta evidente nello schema proposto da Segre:

A s'impossessa di Alatiel

A ne diventa l'amante

(A muore ad opera di B);

B s'impossessa di Alatiel

B ne diventa l'amante

(B muore ad opera di C)

e così via per $\mathrm{C}, \mathrm{D}$ ecc. (150)

Lo struttura è vivacizzata da alcune varianti, come il mancato possesso da parte del marinaio genovese ridotto in fin di vita dalla lotta per la precedenza con il suo complice, o il mancato delitto in altri casi (sostituzione del principe di Morea al marinaio o di Constanzio al duca di Atene).

In tutta la vicenda Alatiel, priva del linguaggio e dunque della possibilità di arginare la sua riduzione a merce da parte degli ammiratori, ostenta una sorta di sonnolenta passività. Il suo cordoglio alla scomparsa del primo dei suoi compagni, ("la donna amaramente e della sua prima sciagura e di questa seconda si dolse molto" 37), facilmente lenito dal nuovo padrone, si ripeterà sbiadito nel passaggio di proprietà successivo ("nuovo cordoglio ... . a far cominciò" 40), per scomparire negli episodi seguenti. Alatiel si comporta come una qualsiasi femmina del mondo animale che si accoppia col maschio vincitore nella lotta per il 
suo possesso per una logica istintiva: anche quando non la si direbbe scontenta di una situazione e di un compagno, la troviamo pronta ad adeguarsi al mutamento di scena, convalidando così il procedere degli uomini che si succedono nella sua vita, che si preoccupano solo dell'eliminazione del rivale e considerano scontato o comunque indifferente il consenso della donna. Non è questa una soluzione sempre vincente nel Decameron: in 4.9 Guiglielmo Rossiglione uccide il rivale Guiglielmo Guardastagno, ma la donna preferirà uccidersi a sua volta piuttosto che sopravvivere all'amato. Mutatis mutandis, ritroviamo lo stesso schema e lo stesso scarto tra la logica dell'autorità e quella della figura sottomessa nella novella di Lisabetta da Messina (4.5). L'eliminazione del rivale non è sempre risolutiva: alla donna è concesso il sottrarsi alla logica che la vuole preda del più forte, anche se lo scarto si traduce nella decisione estrema di togliersi la vita. La storia di Alatiel presenta però una differenza sostanziale rispetto ai due esempi indicati, che è poi la stessa che segna la sua distanza dal romanzo alessandrino che la critica ha indicato come modello parodiato nella novella ${ }^{1}$ : Alatiel non ama nessuno dei suoi possessori. La sua mancanza d'amore sembra speculare a quella dei suoi amanti: come quegli uomini sono tutti attratti nello stesso modo, siano essi principi o mercanti, ${ }^{2}$ da un fantasma senza voce, così Alatiel tutti uguali li considera, preoccupata solo dei vantaggi che dalla permanenza con l'uno invece che con l'altro potrà ottenere. Mentre il romanzo alessandrino narra le tribolazioni di due amanti, in cui il mantenimento della verginità è la prova dell'assenza di cedimenti e di una fede costante, la novella mette in scena un personaggio fin dall'inizio ridotto ad una merce di scambio, nella logica della ragion di stato. Per Alatiel la differenza tra il re del Garbo e uno qualsiasi dei suoi amanti non è che sociale (essere moglie di un re è meglio che essere amica di un castellano), e la verginità (o l'apparenza della stessa) non è che funzionale a quell'obiettivo, nella paura che la merce, trovata difettosa, venga rispedita al mittente. Per questo sposare le logiche altrui per i propri fini, Muscetta (201) considera Alatiel il prototipo dell'Angelica ariostesca, da cui la separa però la mancanza di iniziativa e una certa opacità di atteggiamenti ed intenzioni. Invano ci aspetteremmo da lei un ragionamento serrato e lucido come quello di Angelica che si appresta a chiedere aiuto a Sacripante:

Pur tra quei boschi il ritrovarsi sola le fa pensar di tor costui per guida; che chi ne l'acqua sta fin alla gola, ben è ostinato se mercé non grida.

È una battuta che indica conoscenza delle regole del gioco, ma anche consapevolezza che di un gioco si tratta, e che la parte suggerita dalle circostanze potrà essere abbandonata in tempi migliori. Angelica non è, insomma, la parte che recita: il suo percorso alieno agli ideali cavallereschi culminerà nell'"illogico" 
amore per Medoro. Nella passività di Alatiel, invece, compaiono i termini di una silenziosa acquiescenza, se non una complicità con il mondo dei suoi rapitori, che costituiscono l'altra faccia del suo adeguamento mimetico ad una realtà che la agita ma che non dipende da lei.

Ma chi è, allora, Alatiel? L'assenza del linguaggio si ripercuote non solo sull'esterno, ma anche verso l'interno: non solo Alatiel non dà segni di reazione, ma sembra incapace di definire anche a suo beneficio la natura delle avventure. Non dimentichiamo, del resto, la sua inesperienza: il suo ingresso nel mondo dei sensi la trova priva della parola, vale a dire della possibilità di definire quello che le sta accadendo. L'impotenza linguistica di Alatiel va interpretata nel senso più profondo: il linguaggio così come lei l'ha ricevuto, il linguaggio in cui si è più 0 meno riconosciuta fino al momento del naufragio, non offre definizioni per le vicende di sesso e sangue che la vedono coinvolta. Il naufragio del linguaggio è il naufragio della ragione ${ }^{3}$ : nel momento in cui le sue categorie si rivelano inadeguate, Alatiel vede delinearsi la logica di un discorso in cui lei entra solo in quanto oggetto, incapace percio di formulare tanto una ribellione quanto un consapevole assenso. È per questo che i suoi lamenti alla morte dei primi compagni risultano così fuori luogo: sono i residui del vecchio linguaggio, che esprimerebbe assai bene il lamento della vedova del re del Garbo, ma risulta stonato in quel contesto. La reiterazione, del resto, ne rivela subito l'incongruità.

Sul piano narrativo è di fondamentale importanza notare la coincidenza tra il silenzio che la protagonista osserva per gli uomini che le si accompagnano e quello per il lettore. È questa una coincidenza niente affatto comune se si paragona la novella a quelle, più numerose, la cui riuscita dipende dal fatto che il lettore ne sa di più dei personaggi ed è quindi in grado di apprezzare al meglio le dinamiche linguistiche e strutturali: un esempio canonico è quello della novella di ser Ciappelletto (1.1): la conoscenza da parte del lettore della vera natura del protagonista e l'ignoranza della stessa da parte del confessore è il presupposto per la comprensione della natura ambigua della confessione e in definitiva per la riuscita stessa della novella. In 2.7 siamo invece costretti a sposare il punto di vista dei rapitori di Alatiel, e il volto della donna rimane come offuscato dalla sua stessa bellezza. Ai nostri fini non è tanto importante che Alatiel non parli, quanto che non si parli, che non ci sia dato di assistere al formarsi di una qualche reazione e coscienza. Di qui quel tanto di insoddisfazione da parte del lettore, lasciato col fantasma di questa eroina che si presta ad ogni interpretazione e al tempo stesso le sfugge tutte. Possiamo chiederci con Marcus se sia lecito vedere in Alatiel qualcosa di più di uno schermo per le altrui fantasie, se il protagonista della storia non sia piuttosto il desiderio maschile che mette in scena se stesso e si crea compiacente una larva femminile spersonalizzata e senza voce, pronta ad adeguarsi. ${ }^{4}$ Parafrasando Jacobus (83), potremmo domandarci se c'è davvero una donna in questo testo.

La parabola di Alatiel è dunque quella di una progressiva alienazione dal linguaggio, che si svolge parallela alla riduzione ad oggetto inanimato, fino alla ri- 
soluzione finale. Per Segre la comicità "strutturale" della novella risiede nell'inserzione, nello schema tipico del romanzo alessandrino (promessa di matrimonio-traversie ritardatrici-attuazione del matrimonio), di contatti camali che,

oltre ad essere in contraddizione con un protagonista ideale, dovrebbero escludere ipso facto la possibilità del lieto fine (matrimonio). (154)

Questa interpretazione rende conto però soltanto della comicità per così dire complessiva della novella, presuppone ciò̀ un lettore già esperto del finale, che magari rilegga sulla scorta di quello le tappe avventurose di Alatiel. Queste presentano però, anche a prescindere dall'epilogo, un aspetto comico, legato al meccanismo, apparentemente tutt'altro che divertente, della reificazione della donna.

Il termine "cosa" ricorre spesso nella caratterizzazione di Alatiel, ed è messo direttamente in relazione con la sua incapacità di comunicare in almeno una circostanza, quella che la vede sedere tra il principe di Morea e il suo futuro rivale, il duca di Atene:

non si poté di ragionar con lei prendere piacer, per ciò che essa poco o niente di quella lingua intendeva, per che ciascun lei sì come maravigliosa cosa guardava. (50)

Alatiel è guardata come una cosa perché non è possibile parlarle, perché il desiderio, non potendo sublimarsi ed incanalarsi attraverso la parola, rimane allo stato grezzo. L'assenza di linguaggio di Alatiel si riverbera infatti sui suoi possessori: la parola non è inefficace solo perché non è proferita, ma anche perché non pud essere ascoltata. Privati della parola, i suoi amanti compiono il percorso inverso a quello di Cimone, che l'amore ha "di caprone fatto tornare uomo" (5.1.23). ${ }^{5}$ La comicità risulta allora, come sostiene Savelli, ${ }^{6}$ dalla riduzione ad oggetto di Alatiel, che oggetto non è. L'umanità di Alatiel è implicita nel sorriso di chi legge: un oggetto capace di reazioni, siano pure elementari come quelle di Alatiel, non è più tale, e $\mathrm{i}$ suoi possessori si rivelano colpevoli di un errore di valutazione paradossale che si ritorce contro di loro. La riduzione a cosa di Alatiel da parte dei suoi amanti è tale che, per una sorta di beffardo contrappasso, essi rimangono vittime di una proprietà metafisica degli oggetti: nella parodia del romanzo alessandrino si innesta il tema della pietra stregata, dell'oggetto maledetto che porta disgrazia al suo possessore. Chi si appropria di Alatiel in quanto cosa, sarà punito dall'aspetto reificato di lei. Quando l'aspetto verbale della comunicazione viene ripristinato, l'incantesimo scompare: non solo Antioco non uccide nessuno per averla, ma è anche il primo degli amanti a non morire di morte violenta. Il mercante che gli si sostituisce, anch'egli padrone della lingua della donna, avrà addirittura salva la vita. ${ }^{7}$

Segre nota come la bipartizione tra Alatiel muta e Alatiel parlante si traduca a livello narrativo nella compresenza di due registri stilistici:

Sino all'inizio della prigionia presso Pericone (8-25), e a partire dall'incontro con Antioco (90-120), Alatiel è personaggio nobile, ed è ammantata dallo stile che le 
compete secondo il galateo retorico: autonoma la decisione, dignitosi i gesti, sapienti i discorsi. È invece durante la lunga parentesi delle sue avventure che il Boccaccio si permette di trattarla con stile mediocre, e spesso con la malizia dei doppi sensi: «non avendo mai saputo con che como gli uomini cozzano», 39; «col santo Cresci in man che Iddio ci diè»; «quasi da uguale appetito tirati, cominciatisi a stuzzicare insieme . . . insieme fecero parentado», 89. (153-54)

Il passaggio da un registro all'altro è facilitato e reso più plausibile dal fatto che, anche quando Alatiel è in grado di parlare, i suoi discorsi ci vengono sempre riferiti in maniera indiretta. Ciò rende più drammatiche le due circostanze in cui la voce di Alatiel risuona direttamente. Il ritorno al regno della parola è infatti progressivo, preparato dal passaggio da Osbech ad Antioco, che a differenza degli uomini che l'avevano preceduto conosce la lingua della donna. Il mercante cipriota a cui Alatiel viene affidata da Antioco in punto di morte possiede uguali capacità linguistiche, ma ciò non rompe la consegna al silenzio che Alatiel continua ad osservare sulla pagina. Questa viene meno solo nell'incontro con Antigono, quando la donna può esporre la "sua" storia, la propria interpretazione dei fatti. La bellezza ineluttabile di Alatiel che tutti i vincoli travolge sembra un po' offuscata dalla rispettosa indifferenza di Antigono, qualora non si consideri l'elemento nuovo che è venuto a spezzare l'incantesimo, vale a dire la voce della donna che risuona, non solo per l'interlocutore all'interno della storia (Antigono), ma anche per il lettore, a definire "malvagia" la propria "fortuna". Con il ritorno di Alatiel all'individualità l'elemento comico viene meno: se, dopo il suo nobile discorso, Antigono si preoccupasse solo di caricarla volente o nolente sopra una nave per altri porti, saremmo in piena tragedia. Il lieto fine a questo punto è inevitabile. È, ancora, il ritorno al linguaggio che rende la storia suscettibile di una conclusione: non si vede, altrimenti, come il matrimonio possa salvaguardare Alatiel dal principio ripetitivo (quasi di coazione a ripetere) che ha segnato la sua vicenda fino a quel punto, a meno che non si consideri la perdita della possibilità di comunicazione linguistica e il ritorno alla stessa come i due estremi entro i quali si sviluppa la parabola della donna. L'elemento perturbatore non sarebbe allora tanto la tempesta e il naufragio, quanto la perdita del linguaggio e la conseguente riduzione a spazio bianco aperto alle interpretazioni altrui, ${ }^{8}$ una riduzione che avviene ad un triplice livello.

Il primo è interno alla novella, perché il silenzio della protagonista è la condizione che permette agli uomini di appropriarsene senza scrupoli con ogni mezzo, rispondendo in maniera "fisica" all'invito fisicamente lanciato dalla sua bellezza e interpretato come un'incondizionata disponibilità: quest'aspetto risulta evidente nel caso del secondo possessore, Marato, al quale sembra, "secondo che per gli atti di lei poteva comprendere, essere assai bene della grazia sua [di Alatiel]" (32, corsivo mio).

Il secondo livello è quello della brigata: il commento finale di Panfilo, ("«Bocca basciata non perde ventura, anzi rinnuova come fa la luna»", 122), chiosando con una battuta da commedia una storia dai tragici contorni, sembra 
svelarne un aspetto ma la traspone al livello impersonale del proverbio, mentre la metonimizzazione di Alatiel (corrispondente ad una "bocca basciata") non fa che riproporre e riprodurre il punto di vista dei suoi amanti ma non svela il mistero della donna.

I commenti della brigata sono rimandati come di consueto all'esordio della novella successiva, ma sono, come Alatiel, femminili e senza voce, e tali da richiedere l'autorità superiore dell'autore a decodificarli:

Sospirato fu molto dalle donne per li varii casi della bella donna: ma chi sa che cagione muoveva que' sospiri? Forse v'eran di quelle che non meno per vaghezza di così spesse nozze che per pietà di colei sospiravano. (2.7.2)

C'è dunque un ulteriore schermo, una nuova cautela: le donne non si fanno sorprendere a vagheggiare le ripetute nozze di Alatiel, e il vero motivo dei loro sospiri deve essere introdotto, in maniera dubitativa, da un'autorità esterna. L'interpretazione sovrapposta all'interpretazione complica il quadro più che chiarirlo.

L'elemento discriminante a livello conoscitivo tra la brigata e il terzo livello interpretativo, rappresentato dai lettori, è costituito dalle rubriche, ma sarebbe vano cercare qualche indicazione in quella preposta a questa novella. ${ }^{9}$ Il lettore si trova dunque terzo, in questo processo che possiamo visualizzare come una serie di cerchi concentrici, senza che la prospettiva più ampia si traduca a suo beneficio, ché anzi, nel passaggio tra un livello e l'altro, si ha come un processo di rifrazione per cui i termini della questione risultano "spostati": in modo particolare, l'intervento apparentemente risolutivo dell'autore crea in margine alla direttiva esegetica proposta (e quasi imposta) un cono d'ombra in cui finiscono nella loro complessità irrisolta i significati della vicenda e quell'aspetto tragico che, a meno che non si immagini una sorta di allegro cinismo perlomeno singolare nel Decameron, non sembra potersi annullare per effetto del commento finale di Panfilo.

Alatiel simbolo insieme di fragilità umana e resistenza agli eventi, è stato detto (Baratto 96); "disposta al peggio, ma anche decisa a godersi il meglio" (Muscetta 201); "a superhuman figure; mythic, or at least closely related to a myth . . . a priestess of Eros" (Almansi 125): questo ed altro è stato possibile inscrivere nel silenzio imperturbabile in cui la protagonista si annulla, lasciando al lettore la tentazione ed $\mathrm{i}$ rischi di decifrare un personaggio che non c'è.

Un esempio di segno uguale e contrario è quello della novella di Masetto (3.1), che si finge muto per rassicurare della propria discrezione le monache del convento presso il quale lavora come giardiniere. Un parallelo tra le due vicende sembra suggerire che personaggi maschili e femminili hanno accessi diversi non solo al linguaggio, ma anche al silenzio: Masetto, che sceglie il mutismo come strategia di conquista, è sempre padrone della situazione e può uscirne a suo piacere, mentre i silenzi di Alatiel sono funzionali ai desideri dei suoi possessori e si tradurranno in parola solo per caso.

Un'ultima osservazione riguarda il discorso finale di Alatiel, che sancisce il 
suo ritorno alla legittimità sociale. Ebbene, non è che una parte imparata a memoria, recitata sotto lo sguardo attento del regista (Antigono), quella che Alatiel recita al padre. È poco per farcela conoscere. Forse il linguaggio che la riconsegna al suo rango non le corrisponde più del silenzio con cui si adeguava ai desideri dei suoi amanti: l'ineffabile Alatiel, causa inconsapevole di morti feroci che la sfiorano senza ch'ella d'altro si preoccupi se non della propria sventura, rimane, malgrado tutto, consegnata al suo mistero. ${ }^{10}$

\section{The Johns Hopkins University}

\section{NOTE}

1 Segre analizza gli scarti rispetto al modello del romanzo alessandrino, con riferimento alla definizione di "B. La[vagnini] nell'Enciclopedia Italiana, vol. XXX, p. 78f: «Le vicende di una coppia di amanti che, disgiunti da infinite peripezie, cagionate ora dalla volontà degli uomini, ora dal capriccio o dalla persecuzione della fortuna, riescono a mantenersi reciprocamente fedeli, superando le piu aspre prove, e si vedono alfine, oltre ogni speranza, ricongiunti»" (149 n.).

2 Le strategie impiegate sono però diverse, come mette in luce Mazzacurati (59). Anche per il critico gli amanti, sebbene "minuziosamente differenziati nelle tecniche di conquista", sono "indifferenziati nell'attrazione verso l'oggetto".

3 "A proposito della trasformazione della definizione di uomo da zoon logon echon (animale parlante) ad animal rationale, va detto che noi siamo così avvezzi a pensarci come animali razionali, da aver dimenticato, da non saper più che siamo tali (razionali) in quanto parlanti" (Cazzullo 82).

4 "Alatiel becomes the nameless and selfless partner of pornographic fantasy who makes no emotional demands on her mates and frees them of all moral responsibility for their desires" (Marcus 41).

5 Nel percorso di Cimone il linguaggio svolge un ruolo fondamentale. Nella sua prima apparizione lo troviamo senza "né lettera né costume alcuno, anzi con la voce grossa e deforme e modi più convenienti a bestia che a uomo" (4). Poi, la metamorfosi: Cimone "non solamente le prime lettere appard ma valorosissimo tra i filosofanti divenne ... non solamente la rozza voce e rustica in convenevole e cittadina ridusse, ma di canto divenne maestro e di suono" (18-19).

6 Per la rielaborazione di questo intervento rispetto ad una precedente versione (presentata nel corso della conferenza Gendered Contexts: New Perspectives in Italian Cultural Studies, tenutasi presso la Johns Hopkins University il 16-17 novembre 1990), e soprattutto per la parte relativa alla reificazione di Alatiel, sono infinitamente debitrice a Giulio Savelli, alle sue stimolanti, a volte provocatorie, sempre "centrate", osservazioni. Preziosi commenti e consigli ho ricevuto inoltre da Claude Cazalé-Bérard. Un ringraziamento particolare va al professor Pier Massimo Fomi, cui devo la mia introduzione allo studio del Decameron.

7 Non sappiamo se lo stesso privilegio sia toccato al marinaio genovese superstite: il lettore lo lascia in fin di vita, e la sua sorte, marginale ormai nell'economia della novella, non viene esplicitata.

8 L'interdizione alla comunicazione linguistica del personaggio femminile gioca un ruolo simile nella già citata novella di Zima (5.3). Li l'interpretazione dei silenzi altrui si spinge fino al riempirli con le proprie parole esprimenti corresponsione, secondo una strategia che risulterà vincente.

9 "Il soldano di Babilonia ne manda una sua figliuola a marito al re del Garbo, la quale per 
diversi accidenti in ispazio di quatro anni alle mani di nove uomini perviene in diversi luoghi: ultimamente, restituita al padre per pulcella, ne va al re del Garbo, come prima faceva, per moglie" (2.7.1).

10 Cfr. Mazzacurati $37 \mathrm{n}$.

\section{OPERE CITATE}

Almansi, Guido. The Writer as a Liar. Narrative Technique in the Decameron. London and Boston: Routledge \& Kegan Paul, 1975.

Ariosto, Ludovico. Orlando Furioso. Ed. Lanfranco Caretti. Milano-Napoli: Ricciardi, 1954.

Baratto, Mario. Realtà e stile nel Decameron. Vicenza: Neri Pozza, 1970.

Boccaccio, Giovanni. Decameron. Ed. Vittore Branca. Torino: Einaudi, 1980.

Cavarero, Adriana. "Per una teoria della differenza sessuale". Diotima: il pensiero della differenza sessuale. Milano: La Tartaruga, 1987. 43-79.

Cazzullo, Anna. La verità della parola. Ricerca sui fondamenti filosofici della metafora in Aristotele e nei contemporanei. Milano: Jaca Book, 1987.

Irigaray, Luce. Ce sexe qui n'est pas un. Paris: Minuit, 1977.

Jacobus, Mary. Reading Woman. Essays in Feminist Criticism. New York: Columbia UP, 1986.

Marcus, Millicent J. An Allegory of Form. Literary and Self- Consciousness in the Decameron. Stanford: Anma Libri, 1979.

Mazzacurati, Giancarlo. Forma e ideologia. Napoli: Liguori, 1975.

Muscetta, Carlo. Boccaccio. Bari: Laterza, 1972.

Segre, Cesare. Le strutture e il tempo. Torino: Einaudi, 1974. 